\title{
Perceived stress in relation to life satisfaction and diet quality during the lockdown period of the COVID-19 pandemic, in Greece
}

\author{
V. Costarelli ${ }^{1}$ and M. Michou ${ }^{1}$ \\ ${ }^{1}$ Human Ecology Laboratory, Department Economics and Sustainable Development, Harokopio University, Athens, \\ Greece
}

Mental stress, poor life satisfaction and an unhealthy diet are significantly associated with serious negative health outcomes and behaviors (McNaughton et al., 2015; Palmore \& Luikart, 1972; Perez- Araluce et al., 2021). The COVID-19 outbreak has posed considerable challenges for people's health and life satisfaction. The current study aims to investigate perceived stress levels, in relation to life satisfaction and diet quality in Greece adults, during the lock down period of the COVID-19 pandemic. This cross-sectional, population-based study was conducted in Greece, from the 21st of February to the 3rd of April 2021, during the strict lockdown period of the 3rd COVID-19 wave. A total of 2029 adults (75.3\% women and median age 38 years) participated in an online survey. Participants completed a specially designed questionnaire assessing sociodemographic, anthropometric characteristics, perceived stress levels using the Perceived Stress Scale 14 (PSS-14), life satisfaction levels with the Satisfaction with Life Scale (SWLS) and adherence to Mediterranean Diet (MD) using Mediterranean Diet Assessment Tool (MEDAS). Multiple linear regression analysis was employed.

Twenty six percent of the participants reported very low and low life satisfaction levels and only $11 \%$ reported high and very high levels of life satisfaction. With respect to the adherence to the $\mathrm{MD}, 17.6 \%$ had low adherence, $68.1 \%$ moderate adherence and only $14.2 \%$ had high adherence to the MD. Women reported higher levels of MD adherence $(p=0.037)$ and perceived stress levels $(\mathrm{p}<0.0001)$ than men. Multiple linear regression has shown that women $(b=3.052,95 \%$ CI: $2.381-3.722, p<0.0001)$, younger individuals $(b=-0.109,95 \%$ CI: $-0.136-0.082, p<0.0001)$, obese individuals $(b=0.827,95 \%$ CI: $0.009-1.644, p=0.047)$ compared to normal weight, those with lower levels of life satisfaction $(b=-0.667,95 \% \mathrm{CI}:-0.711--0.622, \mathrm{p}<0.0001)$ and lower adherence to MD ( $b=-0.234,95 \% \mathrm{CI}:-0.377--0.091, \mathrm{p}=0.0001)$ were positively associated with higher levels of perceived stress.

In conclusion, perceived stress seems to be significantly associated with lower life satisfaction and poorer diet, during the lock down period of the pandemic, in Greece.

\section{Acknowledgments}

We would like to thank the participants.

\section{References}

1. Mc Naughton CD, Cawthon C, Kripalani C, et al. (2015). Journal of the American Heart Association 29;4(5), 1-9.

2. Palmore E \& Luikart C (1972). Journal of Health and Social Behavior, 13 (1), 68-80.

3. Perez-Araluce R, Martinez-Gonzalez MA, Fernández-Lázaro CI, et al. (2021). Clin Nutr, S0261- 5614(21)00190-4. 